\title{
A study of exploratory behavior as an index of spatial knowledge in hamsters
}

\author{
BRUNO POUCET, NICOLE CHAPUIS, MADELEINE DURUP, \\ and CATHERINE THINUS-BLANC \\ Institut de Neurophysiologie et Psychophysiologie, C.N.R.S.-I.N.P. 9, Marseille, France
}

\begin{abstract}
This experiment investigated the role of exploration in the formation of maps of the environment. The effects of spatial rearrangement of four familiar objects in an open field on subsequent exploratory behavior were studied in hamsters (Mesocricetus auratus). During two exploratory sessions, four groups of subjects were exposed to objects in a particular spatial relation to each other and to a distal pattern. During a testing session, the control group was exposed to the same situation as during the first two sessions, and the three experimental groups were exposed to various object rearrangements. The hamsters in the experimental groups, but not those in the control group, renewed their exploration of the objects during the testing session, as measured by the number of contacts with the objects and the time spent investigating them. Further analyses of the nature of the reinvestigated objects (i.e., displaced or nondisplaced) support the hypothesis that, through exploration, a long-lasting representation of the environment is built up on the basis of the topological relations among the objects, the overall geometric structure provided by the arrangement of the objects, and the relations between the objects and extra-apparatus landmarks.
\end{abstract}

Following the pioneer studies by Hall (1934), who used the open field to test the effects of unfamiliar environments upon emotionality in rats, many experiments have been concerned with the intensity of exploratory reactions to new stimuli such as congeneric scents and illumination level and environmental complexity (e.g., Schenk, 1979), as well as water, food, or new objects introduced into a familiar environment (e.g., Cowan, 1976). In contrast to the number of experiments focusing upon the effect of the characteristics of new objects on the amount of exploration, few studies have attempted to provide evidence that exploratory reactions are also induced by new spatial arrangements of familiar objects. In one experiment, Wilz and Bolton (1971) allowed gerbils to explore an open field that contained either only one object, or a group of objects in fixed locations. After habituation (i.e., extinction of exploration), a new arrangement of the objects or a change in the location of the single object elicited a renewal of exploration as intense as a totally new situation would have done. Similar results were obtained in other experiments with gerbils (Cheal, 1978; ThinusBlanc \& Ingle, 1985) and rats (Corman, Meyer, \& Meyer, 1967; Corman \& Shafer, 1968; Dember, 1956; Lukaszewska, 1978; Lukaszewska \& Dlawichowska, 1982). Such data strongly suggest that exploratory be-

This research was supported by a grant from The Centre National de la Recherche Scientifique (No. ATP 220). Requests for reprints should be directed to B. Poucet, Département de Psychologie Animale, Institut de Neurophysiologie et Psychophysiologie, C.N.R.S.-I.N.P. 9, 31, chemin Joseph-Aiguier, F-13402 Marseille cedex 9, France. havior involves the gathering of information not only about the qualities of the objects but also about their spatial relationships.

Indirect evidence of this function of exploration is provided by the results obtained in spatial problem solving tests. Maier (1932) and, more recently, Stahl and Ellen (1974), Herrmann, Bahr, Bremner, and Ellen (1982), and Ellen, Parko, Wages, Doherty, and Herrmann (1982) showed that rats need an extensive exploratory experience to solve the "three-table problem," that is, to take the shortcut to the goal.

The renewal of exploration following the rearrangement of familiar objects appears likely to be a valid indicator of the detection of a change. Such a detection implies that the second arrangement is compared with some internal representation or "model" of the previous one. This type of experiment appears to provide a suitable means to obtain evidence concerning the capacities of the animals to integrate and set up spatial relationships likely to be the elements of the "cognitive maps" (Tolman, 1948). From this perspective, exploration is a cognitive behavior because it corresponds to the acquisition of knowledge (an internal representation of space); furthermore, such representations or "schemas" may control and organize, by means of feedback, the collection of further information (Neisser, 1976).

The first aim of the present experiment was to determine whether hamsters, like rats and gerbils, after habituation of exploration of a particular configuration of objects, were able to detect a rearrangement of these objects and to so demonstrate by renewing exploration. The second aim was to investigate, by varying the number and 
type of spatial changes, the nature of the spatial relationships that the hamsters spontaneously established.

The exploratory reactions toward objects in an open field were measured by the number of contacts the animals made with the objects and the total time they spent investigating them. These two measures were assumed to reflect investigation of the objects. A measure of locomotor activity did not appear to provide this information. Although locomotor activity is the usual measure of exploratory level (Berlyne, 1960), it would not allow a fine analysis of exploratory reactions toward spatially welldefined objects. Moreover, locomotor activity can be a misleading measure of exploratory behavior, since certain brain damage such as lesions of the superior colliculus (Dean, Pope, Redgrave, \& Donohoe, 1980) or of the septum (Ellen \& Weston, 1983), induce hyperactivity in rats without the responses being typical of exploration or curiosity.

\section{METHOD}

\section{Subjects}

The subjects were 37 adult naive hamsters (Mesocricetus auratus), 21 males and 16 females, born and reared in the laboratory. They were housed in standard cages with littermates of the same sex. They had food pellets ad lib in their cages, throughout the experiment. Prior to beginning of testing, the animals were assigned randomly either to a control group or to one of the three experimental groups (see experimental design).

\section{Apparatus}

The apparatus was a circular open field, $105 \mathrm{~cm}$ in diameter, with walls made of white cardboard, $35 \mathrm{~cm}$ in height. The floor was covered by a white plastic lining. Four different objects were placed into the open field, as shown in Figure 1. The objects were (A) a glass jar (20 cm in height and $8 \mathrm{~cm}$ in diameter), which contained small objects, such as nails, pieces of paper, and so forth, (B) a copper weight $(8 \mathrm{~cm}$ in height and $6 \mathrm{~cm}$ in diameter), (C) a bowl (4 cm in height and $9 \mathrm{~cm}$ in diameter), and (D) a cup (6 cm in height and $6 \mathrm{~cm}$ in diameter). Objects $C$ and $D$ were placed upside-down so that the animals could not have access to the cavity.

The field was illuminated by four $60-\mathrm{W}$-bulbs that were suspended $3 \mathrm{~m}$ above the field equidistantly from each other. The apparatus was surrounded by white curtains so that the visual environment was homogeneous, except for a striped pattern made of cardboard that was placed as shown in Figure 1. A camera, fixed to the ceiling above the apparatus, was connected to a video recorder and a TV screen, allowing the experimenter to record and observe the behavior out of the sight of the animals.

\section{Experimental Design}

The four groups of hamsters received the same treatment during the initial phases of testing (i.e., during preliminary exploration and the first two sessions of exploration), as shown on the left side of Figure 1, but different treatments during the third and final sessions, as shown on the right side of Figure 1. During the third session, the locations of the four objects remained unchanged for the control group (CONT, $n=10$ ), but the locations of one or two object(s) were modified for the three experimental groups.

The spatial locations of the objects in the apparatus can be described in at least three ways: (1) by the topological relationships among the objects (proximity, connectedness, order; e.g., Object A is between Objects B and D), (2) by the overall geometric structure provided by the arrangement of the objects (e.g., the squareshape arrangement during Sessions 1 and 2 ), and (3) by the spatial relationships of the objects in the apparatus to the extraapparatus landmark (striped pattern).

For the first experimental group (EXP 1,n=8), the location of Object $A$ was changed during the third session so that all three types of changes occurred-the topological relationships between Object A and the other objects, the overall geometric structure, and the relationships between Object $A$ and the extra-apparatus landmark.

For the second experimental group (EXP 2, $n=9$ ), Object A was slightly displaced (about $10 \mathrm{~cm}$ from its original location). In this case, the topological cues were unchanged ( $A$ was still between $B$ and $D$, and faced $C$, which remained near the pattern).

For the subjects of the third experimental group $(\operatorname{EXP} 3, n=10)$, Objects $A$ and $C$ were exchanged during the final session. Thus, only the overall geometric structure was unaffected, since the shape of the arrangement of the four objects was still a square, as it had been during the first two sessions of exploration.

\section{Procedure}

The basic procedure was the same for all the subjects. During a 15-min preliminary exploration phase, each subject was introduced at place $\mathrm{P}$ in the apparatus, which did not contain any object (except for small pieces of hazelnuts spread all over the floor). At the end of this period, during which it was allowed to explore and to eat the food, the subject was returned to its home cage. After this preliminary phase, each animal had three sessions of $15 \mathrm{~min}$ each, during which it was allowed to explore the apparatus with the four objects being present. The first of these three sessions came $10 \mathrm{~min}$ (the time required to clean the floor of the apparatus and to set up the objects) after the preliminary phase. The second exploration session took place between 8 and $14 \mathrm{~h}$ after the first one. The same interval was maintained between the second and the third sessions. Two hamsters of the same sex were tested each day. For one of these two hamsters, the preliminary phase and the first session of exploration began at about $8: 30$ a.m.; the second session began at about $4: 30$ p.m. On the same day; the third session began at about 8:30 a.m. on the following day. For the other subject, the preliminary phase and the first exploratory session began at about 5:00 p.m., the second session at about 9:00 a.m. on the following day, and the third session at about 5:00 p.m. on the same day. The testing of another pair of subjects started on the second day, according to the same principle (one subject began testing in the morning and the other one began in the afternoon). This procedure was used to neutralize any possible effects of circadian rhythms upon exploration. Since there were no differences related to the time of testing, the data were pooled and all the reported results are based on the pooled data.

During the first two sessions of exploration, the objects were arranged as shown on the left side of Figure 1. This arrangement remained the same for the control group during the third session, but the locations of one or two objects were changed during the last session for the three experimental groups, as described in the preceding section (see right side of Figure 1). At the beginning of each session, the subject always entered the apparatus at place $P$ (Figure 1). To neutralize the possible effects of olfactory cues (particularly on the third session), the experimenter used plastic gloves when manipulating the objects and also made a point of touching the objects whose locations were not changed.

\section{Data Collection and Analysis}

The data collected through each session were the number of the contacts the animal made with each object and the time it spent investigating each object by contact. A contact was defined as the subject's snout's actually touching an object. A silent stopwatch was used. The videotape was used for measuring the contact times.

Total number of contacts and the total time spent in contact with the objects during each session were averaged across individuals in each group. A repeated measure analysis of variance for paired comparisons (VAR 3 Program; Rouanet \& Lépine, 1970) was car- 


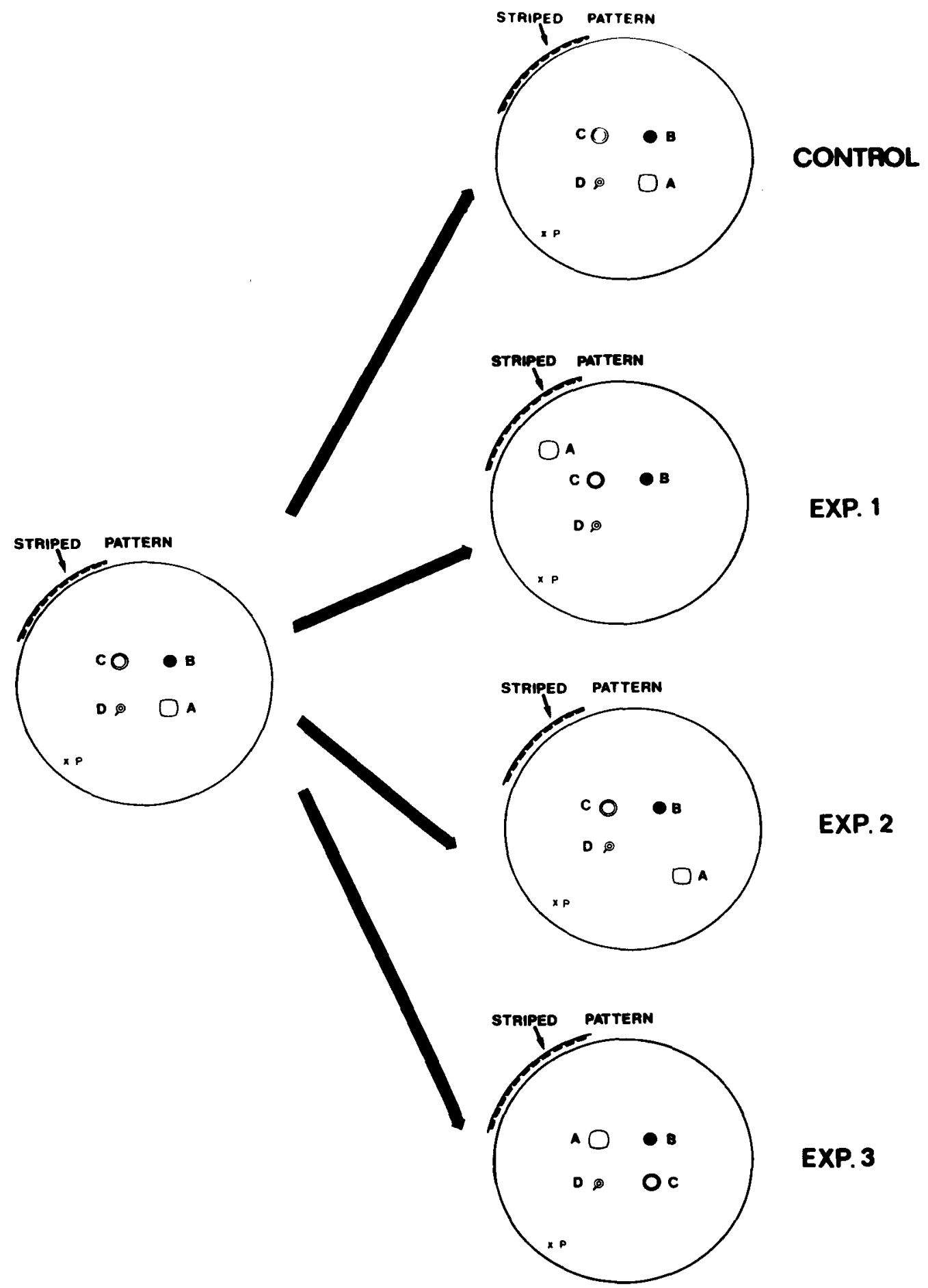

Figure 1. The configuration of objects during Sessions 1 and 2 (common to each group) is shown on the left side; the configuration of objects during Session 3 for each of the four groups is shown on the right side. The place of introduction of the subjects into the apparatus is marked with a $P$.

ried out on the number of contacts and duration of contact. The main factors were group and session.

To ascertain whether exploration during the third session involved displaced objects or nondisplaced objects, or both, another analysis was made which separately processed the data concerning the displaced object(s) and those concerning the nondisplaced objects.
The mean number of contacts per object and per subject and the mean time of exploration per object and per subject were calculated for each class of objects (i.e., displaced or nondisplaced). This calculation was done for the second session which served as a reference level and for the third session as the testing session. Therefore, this treatment was carried out on Object A versus Objects B, 
C, and D for Groups EXP 1 and EXP 2, and on Objects A and C versus Objects B and D for Group EXP 3. Moreover, to get a measure of what occurred in the control group, the same calculation was made on the corresponding objects (although, in this case, there were no displaced objects). Thus, the data from Group CONT were analyzed twice: first by examining the exploration of Object $\mathbf{A}$ versus the exploration of Objects B, C, and D, second, by examining the exploration of Objects $A$ and $C$ versus the exploration of Objects $B$ and $D$. This subdivision provided a reference for the exploration of displaced and nondisplaced objects, respectively, for Groups EXP 1 and EXP 2, on the one hand, and for Group EXP 3, on the other. These two sets of data from the control group will be referred to as CONT $1+2$ for data comparable to those of Groups EXP 1 and EXP 2, and as CONT 3 for data comparable to those of Group EXP 3. It is important to keep in mind that these data, CONT $1+2$ and CONT 3, come from a single group, CONT, but have been arranged in two different ways so that they can be matched with the data from each experimental group.

Two separate analyses of variance (VAR 3 program; Rouanet \& Lépine, 1970) were carried out upon repeated measures of the num- ber of contacts, for the purpose of comparing CONT 1+2, EXP 1 , EXP 2, on the one hand, and CONT 3 and EXP 3, on the other. In addition, the times of contacts were analyzed separately using the same method. In both cases, the main factors studied were group, object (displaced vs. nondisplaced), and session (Sessions 2 and 3).

\section{RESULTS}

\section{Global Exploration}

Number of contacts. Figure 2 shows the mean number of contacts made with the objects by each group as a function of session. There was a decrease in the number of contacts from Session 1 to Session 2 in each group; for the control group, the number of contacts remained stable from Session 2 to Session 3; for the three experimental groups, the number of contacts increased from Session 2 to Session 3. The analysis of variance performed on these data showed that session had a signifi-

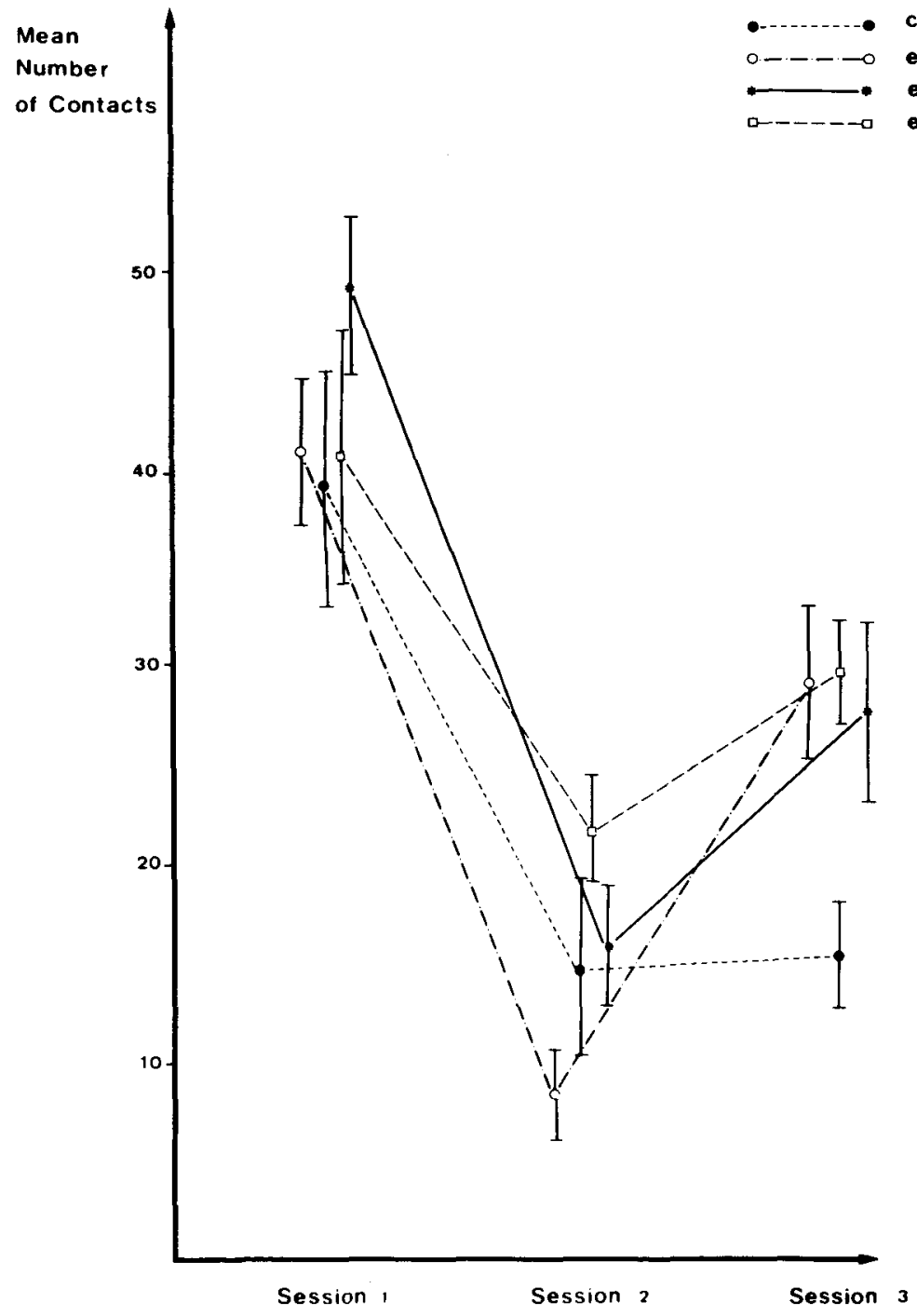

Figure 2. Mean number of contacts with the objects, for the control group and the three experimental groups. (Standard errors are represented by vertical bars.) 
cant effect between Sessions 1 and 2 for all the groups [CONT, $F(1,9)=21.96, p<.01$; EXP $1, F(1,7)=$ $64.57, p<.001$; EXP 2, $F(1,8)=95.34, p<.001$; EXP 3, $F(1,9)=6.88, p<.05]$ and a significant effect between Sessions 2 and 3 for Groups EXP $1[F(1,7)=$ $52.25, p<.01]$, EXP $2[F(1,8)=5.81, p<.05]$, and EXP $3[F(1,9)=21.26, \mathrm{p}<.01]$, but not in the control group $[F(1,9)=.02$, n.s.]. Furthermore, although there was no effect of group during the first two sessions [respectively, $F(3,33)=.67$, n.s., and $F(3,33)=2.53$, n.s.], a statistically significant difference between groups during Session 3 was observed $[F(3,33)=3.96$, $p<.05]$. According to paired comparisons, the three experimental groups explored more than did the control group during the last session [comparison CONT-EXP 1, $F(1,16)=8.59, p<.01$; comparison CONT-EXP 2, $F(1,17)=5.46, p<.05$; comparison CONT-EXP 3, $F(1,18)=13.90, p<.01]$. None of the comparisons among the three experimental groups revealed any differences.
Exploration times. Figure 3 shows the mean duration spent investigating the objects during each session. As in the case of the number of contacts, there was a decrease in the measure for all groups from Session 1 to Session 2; the measure increased for the experimental groups from Session 2 to Session 3, but remained stable for the control group. The analysis of variance found the initial decrease from Session 1 to Session 2 to be statistically significant for each group [CONT, $F(1,9)=12.59, p<$ $.01 ; \operatorname{EXP} 1, F(1,7)=18.05, p<.01 ; \operatorname{EXP} 2, F(1,8)$ $=25.78, p<.001 ; \operatorname{EXP~3,F(1,9)=10.66,p<.01]}$. The following increase between Sessions 2 and 3 was confirmed for Groups EXP 1 and EXP 3 [respectively, $F(1,7)$ $=15.48, p<.01$, and $F(1,9)=13.81, p<.01]$ but not for Group EXP $2[F(1,8)=4.89$, n.s. $]$ or Group CONT $[F(1,9)=.01$, n.s.]. Moreover, there was no effect of group during the first session $[F(3,33)=.89$, n.s. $]$ or the second session $[F(3,33)=.56$, n.s. $]$. In contrast, the difference among groups during the third session was statistically significant $[F(3,33)=4.66, p<$

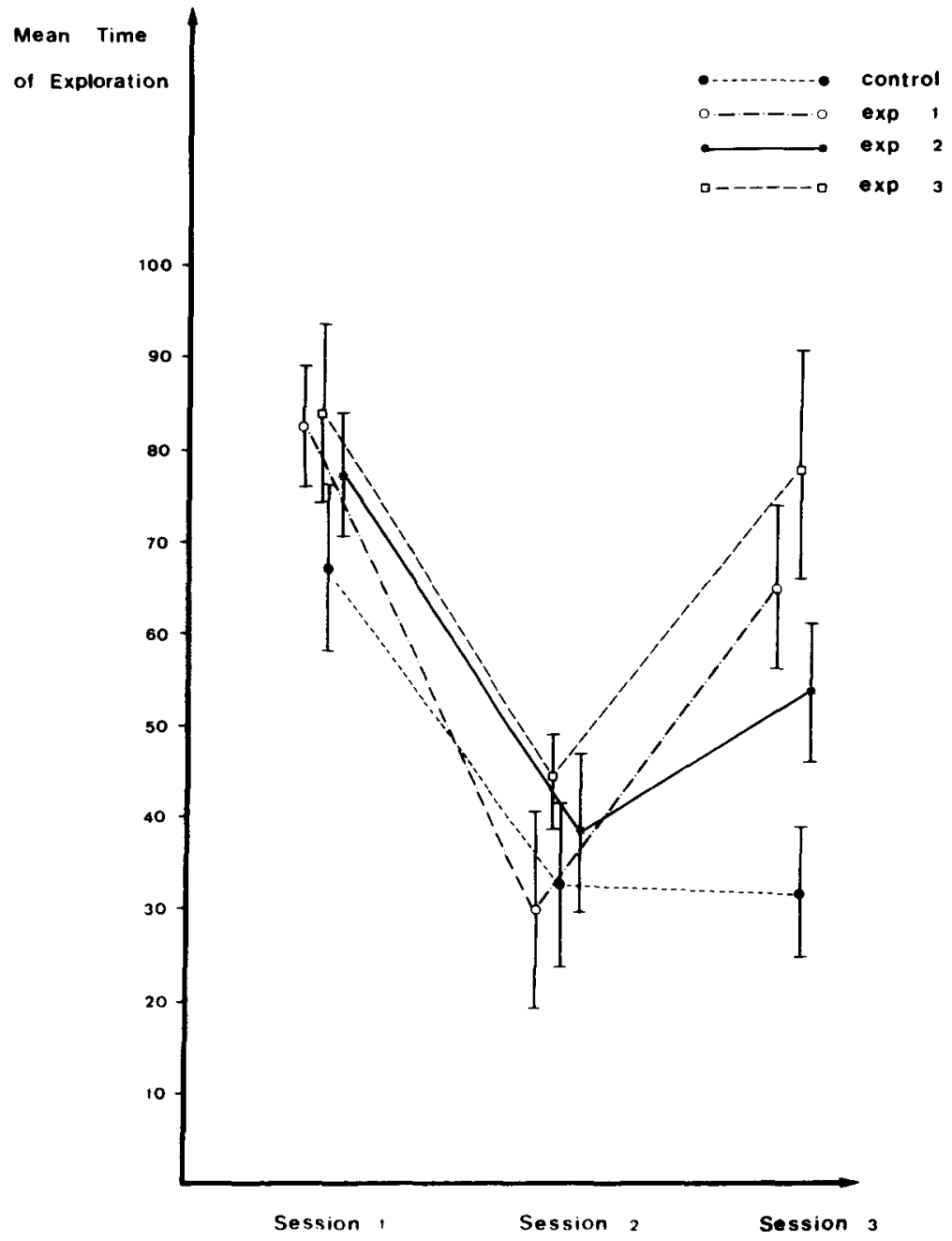

Figure 3. Mean time (seconds) spent investigating the objects, for the control group and the three experimental groups. (Standard errors are represented by vertical bars.) 
.01]. In that respect Groups EXP 1 and EXP 3 explored more than did the control group during Session 3 [comparison CONT-EXP $1, F(1,16)=8.90, p<.01$; comparison CONT-EXP 3, $F(1,18)=10.54, p<.01]$. No statistical difference was found between Groups CONT and EXP 2 during the last session $[F(1,17)=4.25$, n.s. $]$. Furthermore, Group EXP 2 was not statistically different from Groups EXP 1 and EXP 3 in this last session [respectively, $F(1,15)=.96$, n.s., and $F(1,17)=2.70$, n.s.]. The lack of a statistically significant effect of the change introduced during the third session for Group EXP 2 was due to the fact that 2 hamsters, among 9, failed to habituate during Session 2; therefore, the possible effects of the change upon exploration during Session 3 may have been masked by the lack of habituation during the preceding session.

\section{Displaced Versus Nondisplaced \\ Objects Exploration}

To simplifly presentation of the data related to the exploration of the displaced and nondisplaced objects, the differences between the data from Session 3 and those from Session 2 were calculated and then represented in histograms for either the number of contacts with the objects or the time spent to explore them. The statistical tests were made on the raw data, and their results are reported in Figures 4 and 5. If an animal was exploring significantly more during Session 3 than during Session 2 (the actual hypothesis tested), then the difference between Session 3 and Session 2 was significantly above zero, as shown in Figures 4 and 5.
Numbers of contacts. The results for number of contacts are summarized in Figure 4, which shows the differences, N3-N2, between the mean number of contacts during Session 3 and Session 2, with the displaced object(s) and with the nondisplaced objects. In the control group (Figure 4: CONT $1+2$ and CONT 3), there was no increase or decrease in the exploratory reactions toward either object corresponding to those displaced or nondisplaced in the experimental groups, but in all experimental groups there was a significant increase in the exploration of displaced object(s) [EXP 1, $F(1,21)=54.83, p<$ $.001 ; \operatorname{EXP} 2, F(1,24)=6.66 ; p<.05, \operatorname{EXP} 3, F(1,27)$ $=18.18, p<.001$; Figure 4-EXP 1, EXP 2, and EXP 3]. Furthermore, only Group EXP 1 had a significant increase in the number of contacts with the nondisplaced objects $[F(1,21)=10.98, p<.01]$. Finally, subjects in all the experimental groups explored the displaced object(s) more that the nondisplaced ones during the third session [EXP 1, $F(1,24)=20.40, p<.001$; $\operatorname{EXP} 2, F(1,24)=8.70, p<.01 ; \operatorname{EXP} 3, F(1,27)=$ $20.99, p<.001]$, but they showed no preference for these objects during the second session.

Exploration times. Figure 5 shows the differences, $\mathrm{T} 3-\mathrm{T} 2$, between the mean time spent exploring the displaced and nondisplaced objects during Sessions 3 and 2 . The subjects of the control group spent the same amount of time exploring either class of objects during both sessions, but the animals of the three experimental groups increased the duration of investigation of the displaced object(s) [EXP 1, $F(1,21)=17.98, p<.001$; EXP 2, $F(1,24)=8.68, p<.01 ; \operatorname{EXP} 3, F(1,27)=20.62, p<$

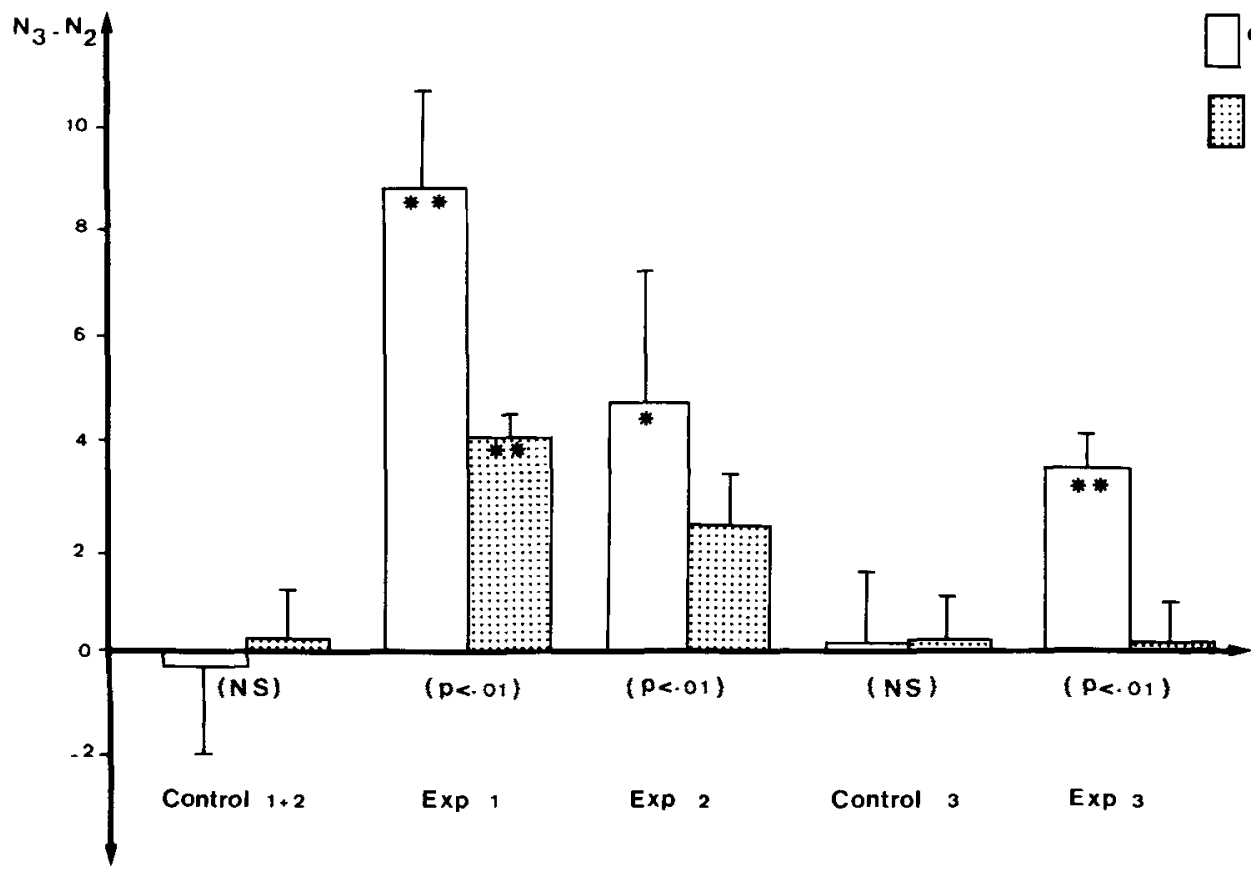

Figure 4. Differences between Sessions 3 and 2 in mean number of contacts with either displaced or nondisplaced objects. Standard errors are represented by vertical bars. Significances of the differences between Sessions 3 and 2 are given by $*(p<.05)$ and $* *(p<.01)$. Significances of the difference between exploration of displaced object(s) and exploration of nondisplaced objects during Session 3 are shown in parentheses. See text. 


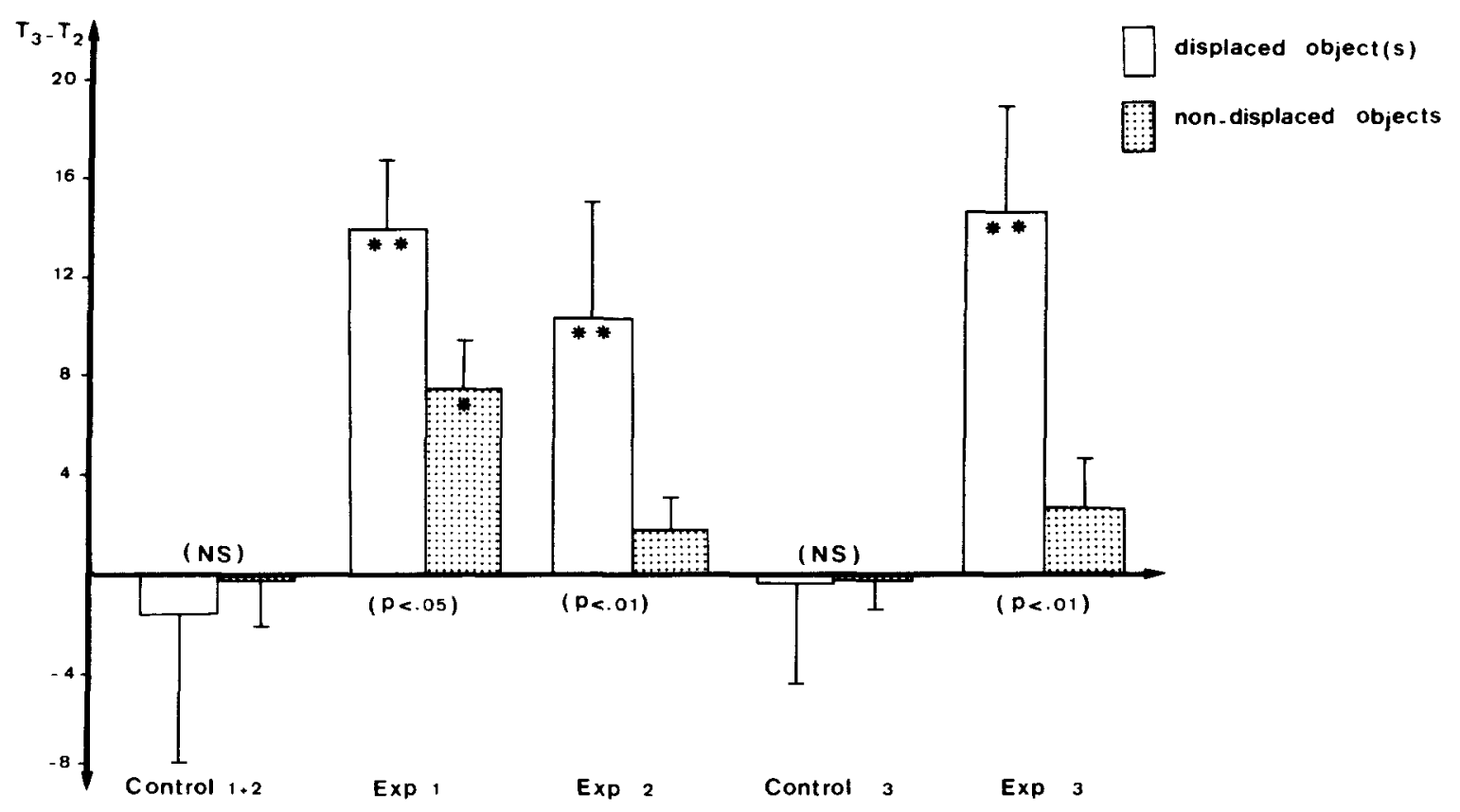

Figure 5. Differences between Sessions 3 and 2 in mean time (seconds) spent investigating either displaced or nondisplaced objects. Standard errors are represented by vertical bars. Significances of the differences between Sessions 3 and 2 are given by $*(p<.05)$ and $* *(p<.01)$. Significances of the differences between exploration of displaced object(s) and nondisplaced objects during Session 3 are shown in parentheses. See text.

$.001]$. Again, the only group showing an increased exploration time of the nondisplaced objects was Group EXP $1[F(1,21)=5.92, p<.05]$. Furthermore, all groups except the control group explored the displaced object(s) more than the nondisplaced ones during the third session [EXP 1, $F(1,21)=7.76, p<.05 ; \operatorname{EXP} 2$, $F(1,24)=17.35, p<.001 ; \operatorname{EXP} 3, F(1,27)=22.03$, $p<.001]$, although there was no difference between the exploration times of the two classes of objects during the second session.

\section{DISCUSSION}

The purpose of the present study was first to determine whether a change in the spatial relationships between objects in an already known environment could induce a renewal of the exploratory behavior of hamsters, and, second, to obtain information about the nature of the spatial changes responsible for this renewal. Four groups of hamsters were subjected to experiments in which different classes of spatial information were manipulated. Exploration was defined by two measures: the number of contacts with the objects and the time spent investigating them. After two 15-min exploratory sessions in a field containing four objects, the subjects of the different groups were placed, for a final exploratory session, in the same field in which either the arrangement of the four objects had not been modified from that used during the first two sessions (control group) or the location(s) of one or two objects had been changed with respect to their initial location(s) (experimental groups). The spatial changes were designed to dissociate the respective roles. of such different spatial cues as the topological relationships between the objects, the overall geometric structure, and the relationships between the objects and the extra-apparatus landmark.

The results showed that all except two of the subjects (from Group EXP 2) habituated (i.e., reduced their exploration) during the first two sessions. Exploration of the control group remained at a very low level during the third session, since the arrangement of objects was unchanged; the three experimental groups, which encountered changes in the arrangement of the objects, renewed their exploration (i.e., increased the number of contacts with the objects and of the time spent exploring them). These results support the hypothesis proposed by several investigators (Dember, 1956; O'Keefe \& Nadel, 1978; Shillito, 1963; Wilz \& Bolton, 1971) that exploration, in addition to familiarizing subjects with object characteristics, plays an important part in the acquisition of knowledge of spatial relations. These results are in agreement with the hypothesis that among the purposes of exploration is that of the building up of environmental maps involving representations that are long-lasting (inasmuch as an average of $11 \mathrm{~h}$ elapsed between Sessions 2 and 3 ). When there are changes in the spatial relationships among objects in a known environment-this knowledge being reflected by habituation and the lack of further exploration as exhibited by the control group-there is a renewal of exploration. This implies that the subjects detected the 
change relative to some internal representation of the initial situation. Exploration would then be undertaken to reduce the animal's "uncertainty" about the environment, and this would lead to an integration of the new spatial relationships. In natural environments, detailed knowledge of the animal's home range, gained by means of exploratory behavior (Shillito, 1963), would have a highly adaptive value, since it would allow the subject to select the best way to get to the food sources or to its nest, according to the circumstances.

Detailed analyses of the nature of the reinvestigated objects (i.e., displaced or nondisplaced) revealed that renewed exploration was directed only toward the displaced objects in Groups EXP 2 and EXP 3, but toward all the objects (displaced and nondisplaced) in Group EXP 1. Thus, although EXP 2 and EXP 3 situations were investigated as partly novel situations, the EXP 1 situation was explored as a totally new one. Whether this difference is due to quantitative differences of the magnitude of spatial change among the three experimental conditions-for example, Object A was much more displaced in EXP 1 than in EXP 2-cannot be determined at present. At least, this effect cannot be interpreted in terms of the number of displaced objects, since in EXP 1 and EXP 2, only one object was moved, but in EXP 3 two objects were moved.

An alternative hypothesis is that the difference between EXP 1, on the one hand, and EXP 2 and EXP 3, on the other, is due to the specific spatial changes involved in each situation. In EXP 1, not only was the relationship between the extra-apparatus landmark and the object affected, but also the topological relationship between it and the other objects and the overall geometric structure was modified. As a result, the animals reinvestigated all of the objects, even though only one object had been moved.

In EXP 2, only the relationship between the extraapparatus landmark and the object and the overall geometric structure were altered. Here the animals reinvestigated only the object that had been moved. In EXP 3, the relationships of the displaced objects to the extraapparatus landmark, as well as the topological relationships among the objects, were modified without change of the overall structure. Again, the animals investigated only the objects that had been moved. From these results, it is clear that both topological relationships and the overall geometric structure are important factors for identifying the spatial relationships among objects. Furthermore, it can be inferred that the animals took into account the spatial relationships between the objects and the extraapparatus landmark, since detection of the displaced objects in EXP 3 could be done only with respect to the landmark location or to the place of introduction of the animal in the experimental field. However, this hypothesis needs further work focusing particularly upon exploration of distal cues (which cannot be directly approached) by means of visual scanning; such studies are currently in progress in our laboratory, using video-actographic techniques
(Lecas \& Dutrieux, 1983) which allow for a recording of exploratory head movements.

\section{REFERENCES}

Berlyne, D. E. (1960). Conflict, arousal and curiosity. New York: McGraw-Hill.

Cheal, M. L. (1978). Stimulus-elicited investigation in the Mongolian gerbil (Meriones unguiculatus). Journal of Biological Psychology, 20, 26-32.

Corman, C. D., Meyer, P. M., \& Meyer, D. R. (1967). Open-field activity and exploration in rats with septal and amygdaloid lesions. Brain Research, 5, 469-476.

Corman, C. D., \& Shafer, J. N. (1968). Open-field activity and exploratory behavior. Psychonomic Science, 13, 55-56.

Cowan, P. E. (1976). The new object reactions of Rattus rattus: The relative importance of various cues. Behavioral Biology, 16, 31-34

Dean, P., Pope, S. G., Redgrave, P., \& Donohoe, T. P. (1980). Superior colliculus lesions in rat abolish exploratory head-dipping in hole-board test. Brain Research, 197, 571-576.

Dember, W. N. (1956). Response by the rat to environmental change. Journal of Comparative \& Physiological Psychology, 49, 93-95.

Ellen, P., Parko, E. M., Wages, C., Doherty, D., \& Herrmann, T. (1982). Spatial problem solving by rats: Exploration and cognitive maps. Learning \& Motivation, 13, 81-94.

Ellen, P., \& Weston, S. (1983). Problem solving in the rat: Septal lesion effects on habituation and perseverative tendencies. Physiological Psychology, 11, 112-118.

HALL, C. S. (1934). Emotional behavior in the rat: I. Defecation and urination as measures of individual differences in emotionality. Joumal of Comparative Psychology, 18, 385-403.

Herrmann, T., Bahr, E., Bremner, B., \& Ellen, P. (1982). Problem solving in the rat: Stay vs. shift solutions on the three-table task. Animal Learning \& Behavior, 10, 39-45.

LeCAS, J. C., \& DutriEUX, G. (1983). Head-movements and actographic recordings in free-moving animals, using computer analysis of video images. Journal of Neuroscience Methods, 9, 357-365

LUKASZEWSKA, I. (1978). The effects of exposure and retention interval on response to environmental change in rats. Acta Neurobiologica Experimentalis, 38, 323-331.

LuKaszewsKa, I. \& DlawichowsKa, E. (1982). How long do rats remember spatial arrangement of visual stimuli? Acta Neurobiologica Experimentalis, 42, 127-133.

MAIER, N. R. F. (1932). A study of orientation in the rat. Joumal of Comparative Psychology, 14, 387-399.

NeISSER, U. (1976). Cognition and reality. San Francisco: Freeman. O'KeEFE, J., \& NADEL, L. (1978). The hippocampus as a cognitive map. London: Oxford University Press.

RouANET, H., \& LÉPINE, D. (1970). Comparisons between treatments in a repeated measurement design; ANOVA and multivariate methods. British Journal of Mathematical \& Statistical Psychology, 23, 146-163.

SCHENK, F. (1979). Comportement exploratoire du Mulot sylvestre. Journal de Psychologie, 2, 157-179.

Shillito, E. E. (1963). Exploratory behaviour in the short-tailed vole. Behaviour, 21, 145-154.

STAHL, J. M., \& ELLEN, P. (1974). Factors in the reasoning performance of the rat. Journal of Comparative \& Physiological Psychology, 87, 598-604.

Thinus-Blanc, C. \& INGLe, D. (1985). Spatial behavior in the gerbil. Journal of Comparative Psychology, 99, 311-315.

Tolman, E. C. (1948). Cognitive maps in rats and men. Psychological Review, 55, 189-208.

WILZ, K. J., \& BolToN, R. L. (1971). Exploratory behavior in response to the spatial rearrangement of visual stimuli. Psychonomic Science, 24, 117-118.

(Manuscript received May 6, 1985; revision accepted for publication December 19, 1985.) 